\title{
Development of body composition reference curves for UK firefighters
}

\author{
G. R. Lessons ${ }^{1}$, D. Bhakta ${ }^{1}$ and H. D. McCarthy ${ }^{1}$ \\ ${ }^{1}$ Public Health Nutrition Research Group, London Metropolitan University, London, N7 8DB, UK
}

Research indicates that UK firefighters suffer prevalence of overweight and obesity exceeding that of the UK general population ${ }^{(1-2)}$. This could hinder both firefighter and public safety due to compromised occupational performance. Aside from increased risk of noncommunicable diseases ${ }^{(3)}$, obese firefighters possibly could have lower levels of cardiorespiratory fitness together with reduced muscular strength compared with healthy colleagues assessed as having a healthy weight ${ }^{(4)}$. To date there are no firefighter-specific body composition References available. Differential misclassification can occur using BMI, a problem which is exacerbated in populations possessing above average skeletal muscle, with firefighters being misclassified in up to thirty three percent of cases ${ }^{(5)}$.

We have now developed firefighter specific body composition References. 320 London Fire Brigade (LFB) male firefighters (mean age: 40, SD: 8.6y) from 28 Greater London fire stations were recruited throughout 2019. Skeletal muscle mass and body fatness were measured via bioelectrical impedance analysis utilising the Tanita MC-780MA system. Appendicular skeletal muscle mass (ASMM $\mathrm{kg}$ ) was calculated by summing the muscle mass of the four limbs for each participant. Centile curves were generated for ASMM and body fat percentage using LMS Chart maker light.

This generated seven age-related reference curves which illustrate the 2nd, 9th, 25th, 50th, 75th, 91st and 98th centiles (Fig. 1).
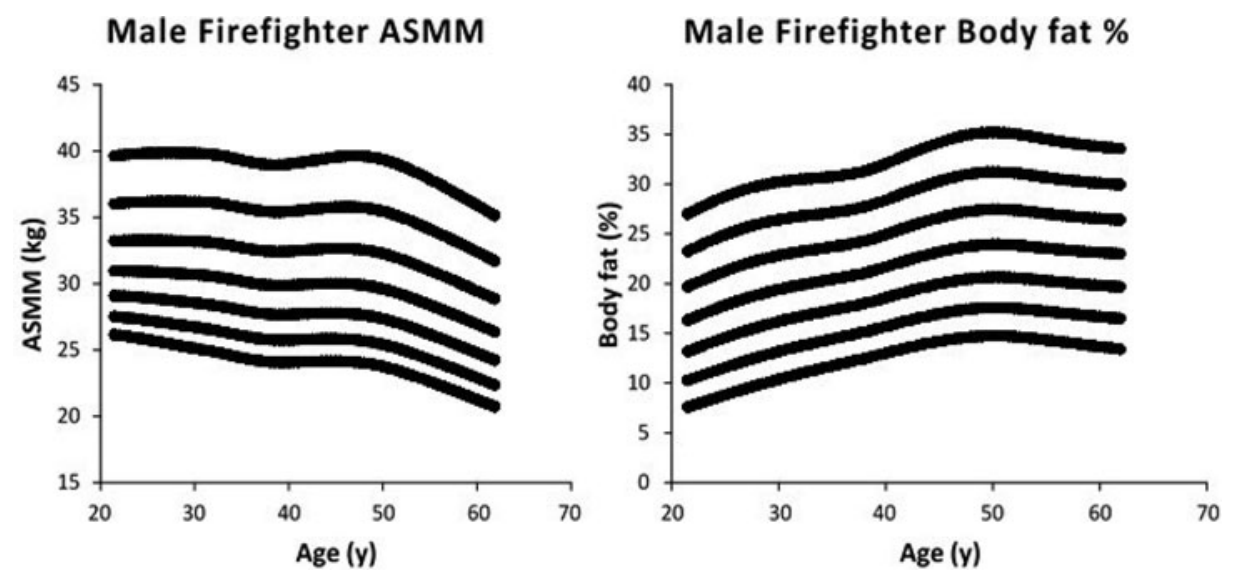

Fig. 1. Male firefighter, age related, appendicular skeletal muscle mass (ASMM) and body fat references.

These centiles offer a novel improvement upon the limitations of BMI, especially when being applied to firefighters who require greater levels of physical fitness and skeletal muscle than the general population. Specific centiles could be assigned to identify age-related low, normal and high ASMM as well as age-related under-fat, normal, over-fat and obese firefighters. They could also be used as education intervention tools to help improve or maintain an optimal body composition. We are currently developing the same References for UK female firefighters.

1. Lessons GR, Bhakta D (2018) Proc Nut Soc 77(OCE1), E21

2. Munir F, Clemes S, Houdmont J et al. (2012) Occup Med 62(5), 362-365.

3. Soteriades ES, Hauser R, Kawachi I et al. (2005) Obes Res 13, 1756-1763.

4. Poston WSC, Haddock CK, Jahnke SA et al. (2011) J Occup Environ Med 53(3), 266-273.

5. Jitnarin N, Poston WSC, Haddock CK et al. (2014) Saf Health Work 5(3), 161-164. 